\title{
Digital Watermarking Algorithm Based on Wavelet Analysis
}

\author{
Xu Qingsong \\ School of Computer and Communication, Changsha University of Science and Technology,Changsha Hunan \\ 410114,China \\ Email: xuqingsong1192@gmail.com
}

\begin{abstract}
With the rapid development of the network and the multimedia technical, how to protect the security of the multimedia informations becomes the popular topic on studies. As a new technique used to protect the copyright of digital productions, the digital watermark technique has drawn extensive attention . A digital watermarking algorithm based on discrete wavelet transform (DWT) was presented according to human visual properties in the paper . Then some attack analyses were given. Experimental results show that the watermarking scheme proposed in this paper is invisible and robust to cropping, and also has good robustness to compression, filtering, and noise adding .
\end{abstract}

Keywords-Digital Watermark;Discrete Wavelet Transform;Human Visual System

\section{INTRODUCTION}

When the traditional encryption techniques can not meet people's needs in the digital multimedia information communication including image, the digital watermarking technology emerges as the times require. In order to not have a detectable change in the carrier image quality while embedded in a larger energy watermark, DWT algorithm combined with the human visual system (HVS) is a hot research topic. A digital watermarking algorithm based on DWT and HVS is presented in the paper .Experimental results show that the algorithm not only has better transparency but also is robust to attacks.

\section{DISCRETE WAVELET TRANSFORM AND HUMAN VISUAL SYSTEM}

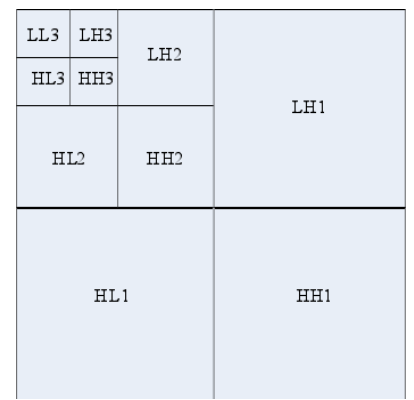

Figue 1 short supporting length, low computation complexity in decomposition and reconstruction and no edge effect.
An image signal's DWT can be regarded as that high-pass and low-pass filtering are used on the column and row of the two-dimension signal respectively. Figure 1 is a schematic diagram for three scales wavelet decomposition. Compared to other compactly supported orthogonal wavelet bases, Haar wavelet base has advantages of

So we select Haar wavelet as wavelet base function of watermark algorithm. In order to make the watermark have good robustness, it is better to embedded the watermarking information into the middle and high frequency coefficients of the wavelet. For example, the third layer's middle and high frequency coefficients of the wavelet. Because they are the results which the second layer's low frequency coefficients are further decomposed into. When watermark is embedded into this frequency band, it has not only the feature that high frequency has little effect on vision, but also the

characteristic that low frequency coefficient is not easy to be modified and emoved. So that it has better imperceptibility and stronger robustness. Lewis and Knowles give a famous visual model to the image compression based on wavelet domain [5] [6] [7].

According to the characteristics of visual cover, watermark 's invisibility is influenced by signal frequency ,background luminance and texture complexity .In the paper, it quantifies the three elements by using this model.

(1) People's vision is more sensitive to the noise in low frequency signal than to the noise in high frequency signal. $\mathrm{F}(\mathrm{l}, \mathrm{O})$ represents the cover factor of the influence on the subgraph by frequency in the $\mathrm{O}$ direction $(\mathrm{O}=\mathrm{LL}, \mathrm{LH}, \mathrm{HL})$ of the $\mathrm{l}$ layer $(\mathrm{l}=1,2,3)$. It can be estimated by the formula (1).

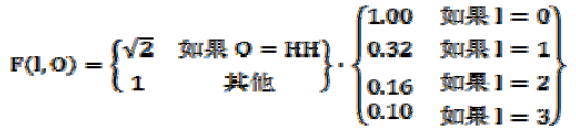

(2)When the image background is lighter, the threshold to invisibility of noise is bigger. The cover factor $\mathrm{Ll}(\mathrm{k}, \mathrm{m})$, which is determined by the brightness of image background, represents the threshold value of coefficient whose spatial locations is $(k, m)$ in the l layer. 
$L(k, m)=3+\frac{1}{256} \sum_{l=0}^{1} \sum_{l=0}^{1} L_{3}\left(i+l+k / 2^{3-1}, j+1+m / 2^{3-1}\right)$

(3)When the image texture is more complicated, its visual threshold value is greater. The cover factor $\mathrm{Tl}(\mathrm{k}, \mathrm{m})$, which is determined by the image Texture, represents the threshold value of coefficient whose spatial locations is $(\mathrm{k}, \mathrm{m})$ in the $\mathrm{l}$ layer.

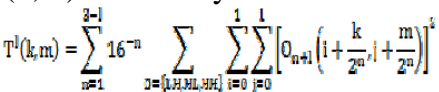

$$
\begin{aligned}
& +\frac{1}{16^{-1-1}} \operatorname{Var}\left\{\left[L_{3}\left(1+x+\frac{k}{2^{3-1}}, 1+y+\frac{m}{2^{2-1}}\right) \mid x=0,1 ; y=0,1\right\}\right.
\end{aligned}
$$

Var represents variance in the formula (3).From the formula (1),(2) and (3), the JND threshold value of wavelet coefficient $\mathrm{q}(\mathrm{k}, \mathrm{m})$ whose spatial locations is $(\mathrm{k}, \mathrm{m})$ and direction is $\mathrm{O}(\mathrm{O}=\mathrm{LH}, \mathrm{HL}, \mathrm{HH})$ in the l layer, can be expressed as $\operatorname{JND}(\mathrm{O} 1(\mathrm{k}, \mathrm{m}))$. It can be estimated by the following

$$
\operatorname{IND}\left(0,[(k, m))=F(1,0) \cdot L^{l}(k, m) \cdot T^{l}(k, m)\right.
$$
formula:

\section{DIGITAL WATERMARKING ALGORITHM}

\section{A. Preprocess Watermark}

Preprocessing the watermark signal can effectively resist the attacks of common low-pass filtering and JPEG compression, and to enhance the robustness and security of the watermarking system. Arnold scrambling transformation has been widely used in digital watermarking because it is simple and has periodicity. For square digital image, Arnold transformation is as follows:

$$
\left[\begin{array}{l}
\mathrm{x}^{\prime} \\
\mathrm{y}^{t}
\end{array}\right]=\left[\begin{array}{cc}
1 & 1 \\
\mathrm{k} & \mathrm{k}+1
\end{array}\right]\left[\begin{array}{l}
\mathrm{x} \\
\mathrm{y}
\end{array}\right] \bmod \mathrm{N}
$$

Where $\mathrm{k}$ is a control parameter and $\mathrm{N}$ is the order of the matrix. (x,y) and (x ', y') represent the position of the watermark image pixels before and after transformation. Assuming that transformation cycle is $\mathrm{M}$, (x, y) will return to the original position after transformation for $\mathrm{M}$ times and it means a image will revert to the original after scrambling for $\mathrm{M}$ times [2]

\section{B. Watermark Embedment}

(1) Use Arnold transformation to scramble binary watermark image Im. And then get the scrambled watermark image Im'. The Scrambling times "N_arnold" is saved as key $\mathrm{K} 1$.

(2) Scan the scrambled watermark image into a one-dimensional sequence for the convenience of being embedded. If the size of the watermark image is $\mathrm{N} * \mathrm{~N}$, the length of the one-dimensional sequence is $\mathrm{N} * \mathrm{~N}$.

(3) $3 \mathrm{~L}+1$ sub-bands will be obtained by using $\mathrm{L}$ layers'wavelet decomposition on the original image. They are $\mathrm{cAL}, \mathrm{cHi}, \mathrm{cVi}$ and $\mathrm{cDi}, \mathrm{i}=1,2, \ldots \mathrm{L}$ where $\mathrm{cHi}+1$,
$\mathrm{cVi}+1$ and $\mathrm{cDi}+1$ are got after decomposition of cAi.

(4) According to the wavelet coefficients obtained after decomposition, the JND threshold value of wavelet coefficient $\mathrm{q}(\mathrm{k}, \mathrm{m})$, whose spatial locations is $(\mathrm{k}, \mathrm{m})$ and direction is $\mathrm{O}(\mathrm{O}=\mathrm{LH}, \mathrm{HL}, \mathrm{HH})$ in the $\mathrm{l}$ layer, can be figured out by the formula (4).

(5) Sort the wavelet coefficients whose direction is $\mathrm{O}(\mathrm{O}=\mathrm{LH}, \mathrm{HL}, \mathrm{HH})$ by the calculated JND value and find the position of $\mathrm{N} * \mathrm{~N}$ wavelet coefficients with the maximum JND value so as to select a corresponding number of wavelet coefficients for embedding. The matrix recording the position of $\mathrm{N} * \mathrm{~N}$ wavelet coefficients with the maximum JND value is stored as key $\mathrm{K} 2$.

(6) According to the formula $\mathrm{W}^{\prime}=\mathrm{W}+\mathrm{a} * \mathrm{~F}$, calculate the value of the changed wavelet coefficients. Where $\mathrm{W}$ ' is the changed wavelet coefficient, "W" is the original wavelet coefficient, " $F$ " is the watermark signal, "a" is the embedding strength.

(7) The inverse transform of the modified wavelet coefficients is implemented in order to obtain the final original image with watermark information[3].

\section{Watermark Extraction}

The extraction process is the inverse process of the above embedding process. As follows:

(1) The original image of the $\mathrm{L}$ layer wavelet decomposition $3 \mathrm{~L}+1$ sub-bands, and these sub-bands are cAL, cHi cVi cDi, i = 1, 2 ... L.

(2) The embedded image of the L layer wavelet decomposition $3 \mathrm{~L}+1$ sub-bands, and these sub-bands are cAL'、cHi'、cVi'、cDi', i=1, 2 ... L.

(3) According to the saved key K2, the watermark was detected in the corresponding position of wavelet coefficients, and using the formula $F=\left(W^{\prime}-W\right) /$ A restore the watermark signal.

(4) Calculating the watermark image scrambling cycle N_arnold, and according to key K1,carry out (N_arnold-K1) times Arnold transform, and recover the binary image watermark signal[4].

\section{IV . EXPERIMENTAL RESULTS AND ANALYSIS}

Usually we use PSNR as the measure of quality of the watermarked image. When getting larger ratio, it represents better quality of the image. The calculation formula about PSNR is as follows:

$$
\text { PSNR }=101 \mathrm{~g} \frac{\mathrm{m} * \mathrm{n} * 255^{2}}{\sum_{\mathrm{m}} \Sigma_{\mathrm{m}}\left(\mathrm{w}(\mathrm{i}, \mathrm{j})-\mathrm{w}^{\prime}(\mathrm{i}, \mathrm{j})\right)^{2}}
$$

In order to quantitatively analyze the similarities between the extracted watermark and the original watermark, it can be described by Normalized Cross-Correlation Function. The formula is as follows:

$$
\mathrm{NC}=\frac{\sum_{\mathrm{m}} \sum_{\mathrm{n}} w\left(\mathrm{~m}_{s} \mathrm{n}\right) * \mathrm{w}^{f}\left(\mathrm{~m}_{s} \mathrm{n}\right)}{\sum_{\mathrm{m}} \sum_{\mathrm{n}}\left(\mathrm{w}\left(\mathrm{m}_{\mathrm{s}} \mathrm{n}\right)\right)^{2}}
$$


Where $\mathrm{w}$ represents the original watermark whose size is $\mathrm{m}^{*} \mathrm{n}$ and $\mathrm{w}^{\prime}$ represents the extracted watermark whose size is $m * n$.

matlab6.5 is used as experiment platform. A standard Lena image of $512 \times 512 \times 8$ is selected as the carrier image and a binary image of $64 \times 64$ is chosen as the original watermark signal. Then conduct simulations according to the proposed embedding and extracting algorithms in the paper. To ensure the watermark's robustness and invisibility, in the HL and LH direction, embedding coefficient " $\mathrm{a}$ " is 15 and in the HH direction , embedding coefficient "a" is $15 * 0.414$. After simulation and calculation, PSNR $=42.7055, \mathrm{NC}=1$.In terms of both subjective visual quality and objective value of PSNR, the watermarked image keeps a excellent quality. Meanwhile similarity between the extracted watermark and the original watermark is also high. The simulation results are shown in Figure 2:

In Order to verify the robustness of the algorithm, then attack the watermarked image. Results are shown in Figure 3:

\section{CONCLUSION}

A digital watermarking algorithm based on discrete wavelet transform (DWT) is presented according to human visual properties in the paper with the following characteristics: (1) The watermark is a meaningful pattern human eye can identify, so it adds vindicability to the watermark. (2)It adds security to the watermark by Arnold scrambling transformation. (3) It adds robustness to the algorithm by embedding the watermarking information into the middle and high frequency coefficients of the wavelet. (4) To ensure good invisibility, choose wavelet coefficients with bigger JND threshold value as embedding location. (5) It adjusts the balance between invisibility and robustness by classifying intermediate frequency domain coefficients and using different embedding strength. Experimental results show that the watermarking scheme proposed in this paper has good robustness to JPEG compression and noise adding. And it is need to be improved in defending rotation attack.

\section{REFERENCES}

[1]Wang,Zhengyou. Li,Zhengxin. Lin,weixin. Liu,Chengcheng. Wang,Wan. Image quality assessment method combination of HVS and similar features[J]. Chinese Journal of Scientific Instrument.pp.6-15,2012,7.

[2] Pan,Lusheng. Grayscale image watermarking technique based on HVS and Wavelet Transform[J]. China packaging industry, pp.20-31, 2012,09

[3] Wei,Yi. Based on wavelet transform image watermarking algorithm simulation and study[J]. China Science and Technology Information, pp,46-65.,2012,08.

[4]A.S.Lewi G.Kowles : Image Compression Using the 2-D Wavelet Transform[J].IEEE Transaetions on Image Proeessing, pp.244-250, 1992,1.

[5]Lewis.A.S. Knowles.G.: Image compression using the 2-D wavelet transform. IEEE Trans. Image Processing, pp.1244-250, 1992.

[6] Watson.A, Yang.G., Solomon,J Villasenor.J:Visibility of wavelet quantization noise. IEEE Trans. on Image Processing, 6(8) pp.1164-1175,1997.

[7]Zhang,Chunsen. Fan,Jinjian. Hu,Pingbo. Wavelet transform and Arnold transform digital watermarking technology[J]. Xi'an University of Science and Technology Journal,pp. 50-74, 2012,01(5).

[8] Li,Yunqi. Assessment of image threshold denoising based on wavelet transform and its effects[J]. Northeast demonstration University Journal , pp.18-60, 2012,01(7).

[9] Mi,xiaozhen, Che,Yu, Dong,Huajun. Digital watermarking algorithm based on discrete wavelet transform and human visual system[J].Dalian Jiao Tong University Journal, pp.12-40, 2012,1(2) .

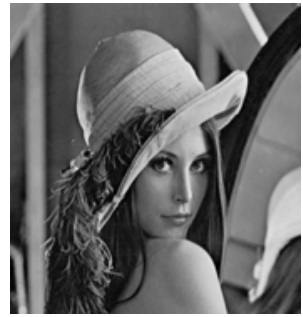

Original image

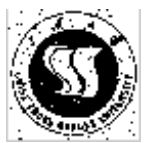

Extracted watermark after compressed

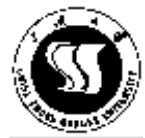

Original watermark

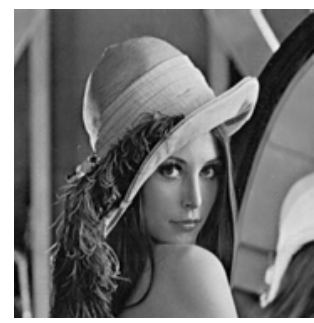

Watermarked image

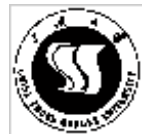

Extracted watermark

Figure 2: simulation results

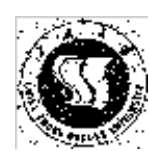

Extracted watermark after noise adding

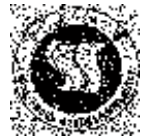

Extracted watermark after filtered

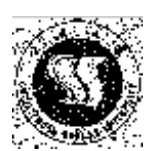

Extracted watermark after cut 